\title{
The Correlation between YAP and RhoA Expression in Prostate and Ovarian Tumor Stroma
}

\author{
Myung-Jin Lee ${ }^{1}$, Dokyeong Kim ${ }^{2 *}$
}

\begin{abstract}
Background and objective: Cancer associated fibroblasts (CAFs) are a mesenchymal cell type found in most solid tumors modulating cancer metastasis by building up and remodeling the extracellular matrix (ECM) structure. We aimed to evaluate the correlation between RhoA and YAP expression in the stroma cells obtained from prostate and ovarian cancer tissues. Methods: We analyzed two microarray datasets obtained from NCBI Gene Expression Omnibus(GEO). The sample type of two datasets was RNA, which is displaying the transcriptome profiling. The tumor stroma of patients with invasive prostate cancer and high-grade serous ovarian cancer were obtained from datasets Independent t-test was used to analyze the differentially expressed $Y A P$ between normal stroma and cancer stroma. In addition, Pearson's correlation was run to analyze the correlation between $Y A P$ and RhoA expressions. Results: In comparison with normal stroma tissues, YAPl was overexpressed in prostate and ovarian cancer stroma tissues (prostate cancer stroma, $\mathrm{p}<0.05$; ovarian cancer stroma, $\mathrm{p}<0.001$ ). Furthermore, a positive correlation was detected between $Y A P$ and RhoA expressions in stroma of both tumor types. This correlation was positively strong in prostate cancer stroma $(\mathrm{R}=0.607)$ and positively weak in ovarian cancer stroma $(\mathrm{R}=0.248)$. Conclusion: We found that $Y A P$ was overexpressed in prostate and ovarian cancer stroma. Furthermore, the correlation between RhoA and $Y A P$ expression suggested that RhoA-YAP signals could physiologically be involved in tumor stroma. Thus, targeting RhoA-YAP may be an intriguing avenue for cancer therapeutics in neoplastic epithelial cells as well as tumor stroma.
\end{abstract}

Keywords: Tumor microenvironment- cancer-associated fibroblasts- YAP- RhoA

Asian Pac J Cancer Prev, 23 (1), 281-285

\section{Introduction}

Over the past decade, the tumor microenvironment (TME) has come to be recognized as an initiator and a promoter of tumorigenesis. Cancer-associated fibroblasts (CAFs) are one of the most dominant components in the TME (Kalluri and Zeisberg, 2006). CAFs are a mesenchymal cells found in most solid tumors modulating cancer metastasis by restructuring and enhancing the extracellular matrix (ECM) (Erdogan and Webb, 2017). Furthermore, CAFs produce growth factors, stimulate tumor angiogenesis, modulate drug access, and respond to cancer therapy (Sahai et al., 2020).

We previously studied the mechanical characteristics and roles of CAFs in cancer progression and found that CAFs led to Ras homolog gene family member A (RhoA) overexpression-mediated cytoskeletal alteration and $Y A P$ (Yes-associated protein) activity, thereby enhancing aggressive phenotypes, including invasiveness and migratory capacity (Kim et al., 2019). Our previous study focused solely on CAFs adjacent to oral squamous cell carcinoma (OSCC). However, most tumors share certain molecular signatures related to cancer, including the sustainment of proliferative signals, evasion of growth suppressors, cell death resistance, angiogenesis induction, and activation of invasion and metastasis (Yuan et al., 2016). For example, the overexpression of HER $2 / \mathrm{neu}$ (ERBB2) has been observed in breast cancer, salivary gland tumors, and uterine and gastric cancers ( $\mathrm{Oh}$ and Bang, 2020). In addition, Wingless-Int (WNT) and transforming growth factor beta (TGF-beta) play critical roles in the regulation of malignant phenotypes in various cancer types (Massague, 2008; Anastas and Moon, 2013). Moreover, specific somatic mutations, such as TP53 and $K R A S$ mutations, can be exhibited across a wide variety of tumor tissues, including lung and pancreas tumors (Schneider et al., 2017). Hence, we aimed to investigate whether the correlation between $Y A P$ and RhoA, which we previously identified in fibroblasts surrounding OSCCs, could be observed in the stroma surrounding different tumor types.

${ }^{1}$ Department of Dental Hygiene, Division of Health Science, Baekseok University, Cheonan, Republic of Korea. ${ }^{2}$ Precision Medicine Research Center, Department of Biomedicine \& Health Sciences, College of Medicine, The Catholic University of Korea, Seoul, Republic of Korea.*For Correspondence: dkkim2908@gmail.com 


\section{Materials and Methods}

NCBI Gene Expression Omnibus (GEO) dataset analysis

We used the publically available data sets in our study downloading the array data for GSE26910 and GSE40595 from NCBI Gene Expression Omnibus(GEO) (http:// www.ncbi.nlm.nih/gov/geo/) database with the platform of the GPL570 [HG-U133_Plus_2] Affymetrix Human Genome U133 Plus 2.0 Array, which were deposited by some researchers (Planche et al., 2011; Yeung et al., 2013). The sample type was RNA, which are displaying the transcriptome expression profiling . The database of GSE26910 contains 24 samples, namely 6 stroma samples surrounding invasive breast primary tumors, 6 matched samples of normal stroma, 6 stroma samples surrounding invasive prostate primary tumors, and 6 matched samples of normal stroma. Among them, we selected 12 samples of prostate cancer stroma and normal prostate stroma. The database of GSE40595 contains 77 samples, including 31 microdissected cancer associated stroma samples, 32 epithelial tumor samples from high grade serous ovarian cancer patients, 8 microdissected normal ovarian stroma samples, and 6 ovarian surface epithelium samples. We selected 39 samples from normal stroma samples and cancer associated stroma samples.

\section{Statistical analysis}

All statistical analyses were done by using SPSS version 20 (SPSS Inc., Chicago, IL, USA). For GEO datasets analysis, independent t-test was used to analyze the difference in specific gene expression between normal stroma and cancer stroma. In addition, Pearson's correlation coefficient was used to analyze the correlation between specific genes.

\section{Results}

Difference between normal stroma and tumor stroma regarding YAP expression

We first investigated differentially expressed $Y A P$

(a)

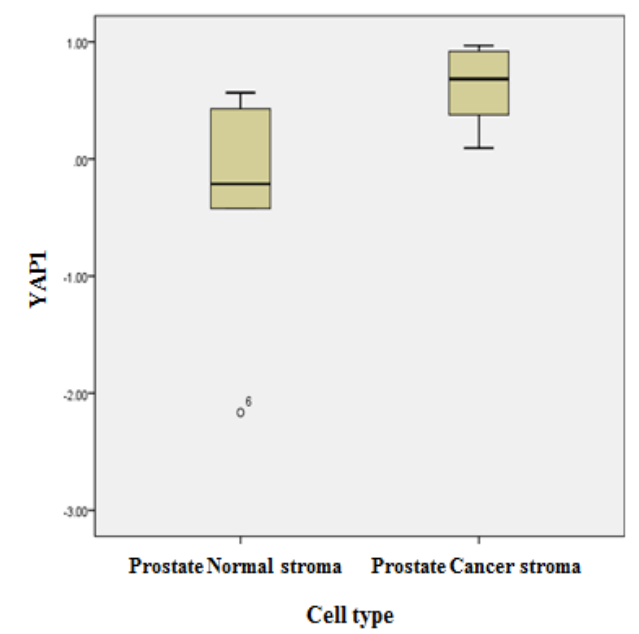

gene between normal stroma and tumor stroma by using publically available data sets from NCBI. We selected two data sets. The first data set included prostate tumor stroma and normal stroma of patients with invasive prostate cancer $(n=6)$. The second data set involved ovarian cancer stroma $(n=31)$ of high-grade serous ovarian cancer patients and normal stroma of healthy individuals $(n=8)$. All intensity values were normalized to log base 2 values. In comparison with normal stroma of prostate and ovarian cancer, the overexpression of $Y A P 1$ was observed in both cancer stroma tissues (Figure 1A and 1B).

The correlation between YAP and RhoA expression in tumor stroma

RhoA is upstream of the YAP transcriptional factor and contributes to $Y A P$ distribution in CAFs surrounding OSCC cells, suggesting that $Y A P$ expression is correlated with $R h o A$ expression. To verify this correlation in stroma surrounding other tumor types, we analyzed the correlation between RhoA and YAP expressions in prostate and ovarian cancer stroma. The positive correlation between YAP and RhoA was identified in the stroma of both tumor types (Figure 2A and 2B). Prostate cancer stroma showed a strong positive correlation $(\mathrm{R}=0.607)$ (Figure $2 \mathrm{~A})$, and ovarian cancer stroma showed a weak positive correlation $(\mathrm{R}=0.248)$ (Figure 2B).

\section{Discussion}

Cancer studies have primarily focused on neoplastic epithelial cells (Dagogo-Jack and Shaw, 2018). However, increasing evidence suggests that the TME plays a critical role in tumor initiation, progression, and therapeutic resistance (Sun, 2016). CAFs constitute a large proportion of the TME; therefore, CAF-targeted cancer therapies are extensively being explored. For CAF-targeting strategies, several CAF markers, such as smooth muscle actin ( $\alpha$-SMA), fibroblast activation protein (FAP), caveolin-1 (cav-1), and platelet-derived growth factor (PDGF), have been studied in the past years (Chen and Song,

(b)

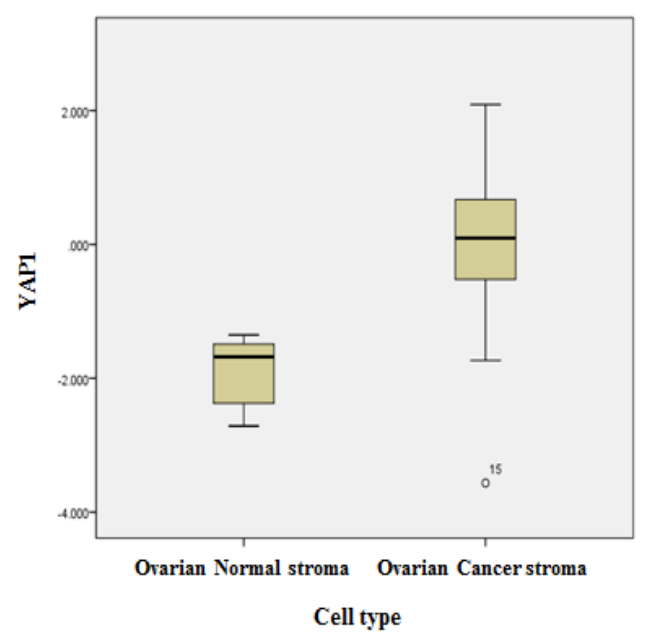

Figure 1. YAP Expression in Cancer Stroma Obtained from Prostate and Ovarian Patients. a, The samples were acquired from prostate cancer stroma and normal stroma in GDS4114 (YAP1, $\left.{ }^{*} \mathrm{p}=0.047\right) ; \mathrm{b}$, The samples were acquired from ovarian cancer stroma and normal stroma in GSE40595. (YAP1, *** $\mathrm{p}=0.000)$ 
(a)

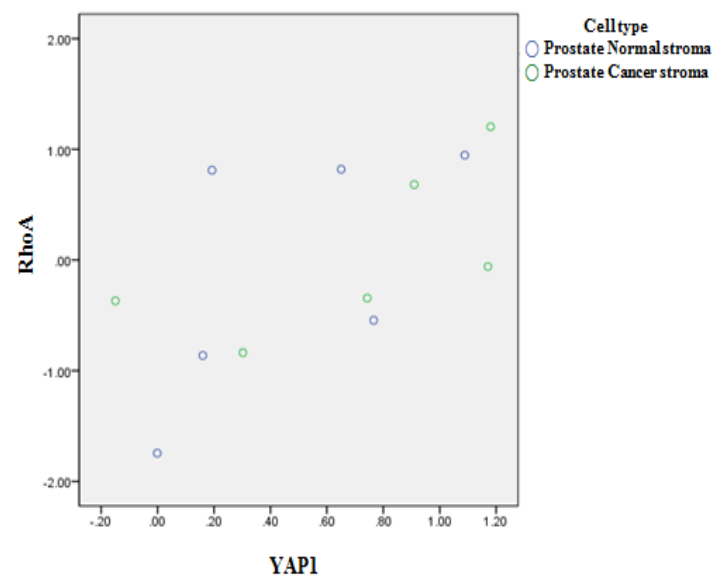

(b)

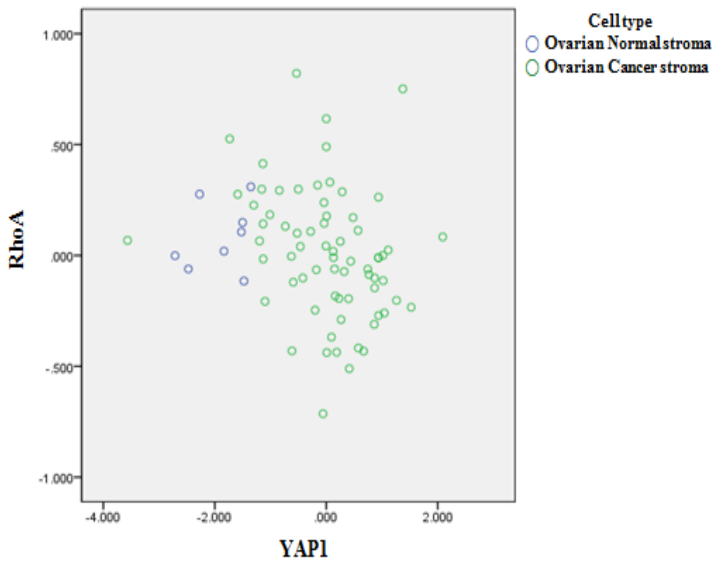

Figure 2. The Correlation between $Y A P$ and RhoA Expression in Normal Stroma and Cancer Stroma. a, The correlation between $Y A P$ and $R h o A$ in normal stroma and prostate cancer stroma (Pearson correlation, $\mathrm{R}=0.067$ (strong positive $(+)$ correlation), ${ }^{*} \mathrm{p}=0.036$; $\mathrm{b}$, The correlation between $Y A P$ and $R h o A$ in normal stroma and ovarian cancer stroma in GSE40595 (Pearson correlation, $\mathrm{R}=0.248$ (weak positive $(+)$ correlation), ${ }^{*} \mathrm{p}=0.029$ ).

2019; Biffi and Tuveson, 2021). However, ubiquitous CAF-targeting agents remain unclear because CAFs have heterogeneous subtypes. CAFs can be derived from numerous cellular sources adjacent to cancer cells, including normal fibroblasts, bone marrow stem cells, and mesenchymal cells transited from epithelial cells (epithelial-mesenchymal transition; EMT) (Chen and Song, 2019; Mhaidly and Mechta-Grigoriou, 2020). The precise origin of CAFs has not yet fully elucidated, implying that the heterogeneity of CAFs is rooted in the diverse cellular subsets. Moreover, CAFs have the phenotypical heterogeneity that activated and senescent phenotypes. Myofibroblasts are considered to be a subset of activated fibroblasts that have contractile and secretory profiles such as alpha-1 type I collagen (COL1A1), interleukin (IL)-1 family member (IL-1 $\alpha$ ), IL-6 $\alpha$, and C-X-C Motif Chemokine Ligand 1 (CXCL1)/growthregulated oncogene (GRO) $-\alpha$, contributing to tissue repair during wound healing and cancer development (Kalluri and Zeisberg, 2006; Bae et al., 2014). Conversely, the senescent phenotype of CAFs is observed adjacent to cancer cells and can secret a senescence-associated secretory protein (SASP) and exacerbate cancer progression, including cancer cell invasion and migration (Wang et al., 2017; Kim et al., 2018a). In addition to phenotypic heterogeneity, functional heterogeneity of CAFs has also been reported. CAFs have historically been considered as tumor-promoting components that enhance cancer cell proliferation, angiogenesis, and metastasis (Erez et al., 2010; Benyahia et al., 2017). However, some studies have been reported that CAFs might have tumor-restraining functions (Biffi and Tuveson, 2021). Stroma cells can reduce neoplastic epithelial cell growth through Hedgehog pathway activity in pancreatic ductal adenocarcinoma (Lee et al., 2014; Rhim et al., 2014). Moreover, Meflin, which is glycosylphosphatidyl inositol (GPI)-anchored protein, suppresses tumor growth in CAFs (Mizutani et al., 2019). As CAFs have the molecular, phenotypic, and functional heterogeneity, targeting specific CAFs is the most challenging aspect. Thus, we previously focused on the common physical characteristics of CAFs, regardless of their diverse cellular origin and their functional and phenotypic heterogeneity. CAFs have traditionally shown higher contractility through ECM remodeling. Moreover, this contractility has been previously reported to aid differentiation between CAFs and normal fibroblasts (Kim et al., 2019; Nurmik et al., 2020). Thus, our findings suggested that more specific markers for tumor stroma may be associated with the physical characteristic of CAFs, YAP overexpression, and RhoA expression .

YAP and TAZ (transcriptional co-activator with WW and PDZ domains; WWTR1) interact with TEAD-family DNA-binding transcription factors. Their interaction influences tissue homeostasis, regeneration, and organ size, and can be responded to Hippo signaling, Wnt, and G-protein coupled receptors (GRCR) (Zanconato et al., 2016). In a wide variety of human cancers, $Y A P$ is thought to be a major contributor to tumorigenesis (Zanconato et al., 2016; Thompson, 2020). For example, YAP overexpression is associated with poor clinical outcomes in ovarian cancer (He et al., 2015a), cervical cancer (He et al., 2015b), and liver cancer (Liu et al., 2010). Moreover, $Y A P / T A Z$ functions as an executer by regulating ECM elasticity, cell shape, and cytoskeletal forces, thereby remodeling the metastatic environment in cancer cells (Dupont et al., 2011; Yu et al., 2015). Consistent with these findings, CAFs showed that YAP expression was regulated by the conformation and tension of the actin cytoskeleton, as the downstream effector of Rho GTPase pathways (Aragona et al., 2013; Calvo et al., 2013; Kim et al., 2019). In a nutshell, these results indicated that $Y A P$ was a core factor in the mechanical regulation of both neoplastic epithelial cells and stroma cells. Furthermore, RhoA, which is a member of the Rho GTPase subfamily proteins, including $R h o A, C d c 42$, and $R a c 1 / 2$, is required for $Y A P / T A Z$ activation according to a previous study (Kim et al., 2018b). Rho GTPases are well known in diverse biological processes, including cytoskeleton regulation, cell adhesion, and cell migration (Haga and Ridley, 2016). 
Among the Rho GTPases, RhoA is involved in tumor progression and metastasis. The overexpression and activity of RhoA are observed in many malignant tumors, such as bladder cancer, liver cancer, and gastric tumors (Gomez del Pulgar et al., 2005). Additionally, RhoA can influence the cell cycle checkpoint and regulate cancer cell survival in gastric cancer cells (Zhang et al., 2009). Thus, RhoA has been considered as a promising target for anti-cancer therapy.

Our study revealed that RhoA-YAP could be a promising therapeutic target. However, our study faced with some limitations. One major limitation was that we focused on YAP expression, not TAZ expression. However, $T A Z$ is a paralog co-activator of $Y A P$ and functions as a downstream effector of the mechanical signaling via the RhoA-ROCK pathway, indicating that $T A Z$ along with $Y A P$ could be crucial cancer therapy target. Moreover, we investigated the expression and correlation between YAP and RhoA in two tumor types, namely prostate and ovarian cancers. To generalize the ubiquitous CAFs markers from a physical point of view, further studies are needed to investigate this gene expression and its correlation with tumor stroma surrounding diverse tumor types. We found that $Y A P$ was overexpressed in the stroma adjacent to different tumor types, such as prostate and ovarian cancers, as well as OSCC. A correlation between RhoA and YAP wasalso been observed in both cancers. Thus, targeting RhoA-YAP signals in CAFs and neoplastic epithelium might be a comprehensive therapeutic approach in prostate and ovarian cancers aw well as OSCC, restricting the formation of a tumor-promoting microenvironment.

\section{Author Contribution Statement}

Conceptualization: D.K. and M.-J.L.; Design: M.J.L.; Literature search: D.K.; Data acquisition: D.K.; Data analysis: M.-J.L.; Manuscript preparation: D.K.; Manuscript editing: D.K. and M.-J.L. All authors reviewed the results approved the final version of the manuscript.

\section{Acknowledgements}

I mistook the this research is part of a $\mathrm{PhD}$ thesis (Dokyeong Kim) approved by Yonsei. However, I identified that the results are not involved in $\mathrm{my} \mathrm{PhD}$ thesis.

\section{Funding Statement}

This study was supported by Basic Science Research Program through the National Research Foundation of Korea(NRF) funded by the Ministry of Education (2021R1I1A1A01045571).

\section{Any conflict of interest}

The authors have no conflict of interest to declare.

\section{Ethical issues}

Not applicable. The data for this study was obtained from public database registered in NCBI.

\section{Availability of data}

All datasets leading to the results of the study are available by the corresponding author on reasonable request.

\section{References}

Anastas JN, Moon RT (2013). WNT signalling pathways as therapeutic targets in cancer. Nat Rev Cancer, 13, 11-26.

Aragona M, Panciera T, Manfrin A, et al (2013). A mechanical checkpoint controls multicellular growth through YAP/TAZ regulation by actin-processing factors. Cell, 154, 1047-59.

Bae JY, Kim EK, Yang DH, et al (2014). Reciprocal interaction between carcinoma-associated fibroblasts and squamous carcinoma cells through interleukin-1alpha induces cancer progression. Neoplasia, 16, 928-38.

Benyahia Z, Dussault N, Cayol M, et al (2017). Stromal fibroblasts present in breast carcinomas promote tumor growth and angiogenesis through adrenomedullin secretion. Oncotarget, 8, 15744-62.

Biffi G, Tuveson DA (2021). Diversity and biology of cancerassociated fibroblasts. Physiol Rev, 101, 147-76.

Calvo F, Ege N, Grande-Garcia A, et al (2013). Mechanotransduction and $Y A P$-dependent matrix remodelling is required for the generation and maintenance of cancer-associated fibroblasts. Nat Cell Biol, 15, 637-46.

Chen X, Song E (2019). Turning foes to friends: targeting cancerassociated fibroblasts. Nat Rev Drug Discov, 18, 99-115.

Dagogo-Jack I, Shaw AT (2018). Tumour heterogeneity and resistance to cancer therapies. Nat Rev Clin Oncol, 15, 81-94.

Dupont S, Morsut L, Aragona M, et al (2011). Role of YAP/TAZ in mechanotransduction. Nature, 474, 179-83.

Erdogan B, Webb DJ (2017). Cancer-associated fibroblasts modulate growth factor signaling and extracellular matrix remodeling to regulate tumor metastasis. Biochem Soc Trans, 45, 229-36.

Erez N, Truitt M, Olson P, et al (2010). Cancer-associated fibroblasts are activated in incipient neoplasia to orchestrate Tumor-Promoting Inflammation in an NF-kappaBDependent Manner. Cancer Cell, 17, 135-47.

Gomez del Pulgar T, Benitah SA, Valeron PF, et al (2005). Rho GTPase expression in tumourigenesis: evidence for a significant link. Bioessays, 27, 602-13.

Haga RB, Ridley AJ (2016). Rho GTPases: Regulation and roles in cancer cell biology. Small GTPases, 7, 207-21.

He C, Lv X, Hua G, et al (2015a). YAP forms autocrine loops with the ERBB pathway to regulate ovarian cancer initiation and progression. Oncogene, 34, 6040-54.

He C, Mao D, Hua G, et al (2015b). The Hippo/YAP pathway interacts with EGFR signaling and HPV oncoproteins to regulate cervical cancer progression. EMBO Mol Med, 7, 1426-49.

Kalluri R, Zeisberg M (2006). Fibroblasts in cancer. Nat Rev Cancer, 6, 392-401.

Kim DK, Kim EK, Jung DW, et al (2019). Cytoskeletal alteration modulates cancer cell invasion through RhoA-YAP signaling in stromal fibroblasts. PLoS One, 14, e0214553.

Kim EK, Moon S, Kim DK, et al (2018a). CXCL1 induces senescence of cancer-associated fibroblasts via autocrine loops in oral squamous cell carcinoma. PLoS One, 13, e0188847.

Kim JG, Islam R, Cho JY, et al (2018b). Regulation of RhoA GTPase and various transcription factors in the RhoA 
pathway. J Cell Physiol, 233, 6381-92.

Lee JJ, Perera RM, Wang H, et al (2014). Stromal response to Hedgehog signaling restrains pancreatic cancer progression. Proc Natl Acad Sci U S A, 111, E3091-100.

Liu AM, Xu MZ, Chen J, et al (2010). Targeting YAP and Hippo signaling pathway in liver cancer. Expert Opin Ther Targets, 14, 855-68.

Massague J (2008). TGFbeta in Cancer. Cell, 134, 215-30.

Mhaidly R, Mechta-Grigoriou F (2020). Fibroblast heterogeneity in tumor micro-environment: Role in immunosuppression and new therapies. Semin Immunol, 48, 101417.

Mizutani Y, Kobayashi H, Iida T, et al (2019). MeflinPositive Cancer-Associated Fibroblasts Inhibit Pancreatic Carcinogenesis. Cancer Res, 79, 5367-81.

Nurmik M, Ullmann P, Rodriguez F, et al (2020). In search of definitions: Cancer-associated fibroblasts and their markers. Int J Cancer, 146, 895-905.

Oh DY, Bang YJ (2020). HER2-targeted therapies - a role beyond breast cancer. Nat Rev Clin Oncol, 17, 33-48.

Planche A, Bacac M, Provero P, et al (2011). Identification of prognostic molecular features in the reactive stroma of human breast and prostate cancer. PLoS One, 6, e18640.

Rhim AD, Oberstein PE, Thomas DH, et al (2014). Stromal elements act to restrain, rather than support, pancreatic ductal adenocarcinoma. Cancer Cell, 25, 735-47.

Sahai E, Astsaturov I, Cukierman E, et al (2020). A framework for advancing our understanding of cancer-associated fibroblasts. Nat Rev Cancer, 20, 174-86.

Schneider G, Schmidt-Supprian M, Rad R, et al (2017). Tissuespecific tumorigenesis: context matters. Nat Rev Cancer, 17, 239-53.

Sun Y (2016). Tumor microenvironment and cancer therapy resistance. Cancer Lett, 380, 205-15.

Thompson BJ (2020). YAP/TAZ: Drivers of Tumor Growth, Metastasis, and Resistance to Therapy. Bioessays, 42, e1900162.

Wang T, Notta F, Navab R, et al (2017). Senescent CarcinomaAssociated Fibroblasts Upregulate IL8 to Enhance Prometastatic Phenotypes. Mol Cancer Res, 15, 3-14.

Yeung TL, Leung CS, Wong KK, et al (2013). TGF-beta modulates ovarian cancer invasion by upregulating CAFderived versican in the tumor microenvironment. Cancer Res, 73, 5016-28.

Yu FX, Zhao B, Guan KL (2015). Hippo Pathway in Organ Size Control, Tissue Homeostasis, and Cancer. Cell, 163, 811-28.

Yuan Y, Jiang YC, Sun CK, et al (2016). Role of the tumor microenvironment in tumor progression and the clinical applications (Review). Oncol Rep, 35, 2499-515.

Zanconato F, Cordenonsi M, Piccolo S (2016). YAP/TAZ at the Roots of Cancer. Cancer Cell, 29, 783-803.

Zhang S, Tang Q, Xu F, et al (2009). RhoA regulates G1-S progression of gastric cancer cells by modulation of multiple INK4 family tumor suppressors. Mol Cancer Res, 7, 570-80.

\section{cc) (i) (3)}

This work is licensed under a Creative Commons Attribution-

Non Commercial 4.0 International License. 